\title{
Factoring the strong CP problem
}

\author{
Prateek Agrawal $^{a}$ and Kiel Howe ${ }^{b}$ \\ ${ }^{a}$ Department of Physics, Harvard University, \\ Cambridge, MA 02138, U.S.A. \\ ${ }^{b}$ Fermi National Accelerator Laboratory, \\ Batavia IL 60510, U.S.A. \\ E-mail: prateek@fnal.gov, khowe@fnal.gov
}

AbSTRACT: We present a new mechanism to solve the strong CP problem using $N \geq 2$ axions, each dynamically relaxing part of the $\bar{\theta}$ parameter. At high energies $M \gg \Lambda_{\mathrm{QCD}}$ the $\mathrm{SU}(3)_{c}$ group becomes the diagonal subgroup of an $\mathrm{SU}(3)^{N}$ gauge group, and the non-perturbative effects in each individual SU(3) factor generate a potential for the corresponding axion. The vacuum is naturally aligned to ensure $\bar{\theta}=0$ at low energies, and the masses of these axions can be much larger than for the standard QCD axion. This mechanism avoids the introduction of a discrete $Z_{2}$ symmetry and associated 'mirror' copies of the SM fermions, and also avoids the introduction and stabilization of new light colored states to modify the running of the QCD gauge coupling found in other heavy axion models. This strengthens the motivation for axion-like particles solving the strong CP problem at points beyond the standard QCD axion curve in the $\left(m_{a}, f_{a}\right)$ plane.

Keywords: Anomalies in Field and String Theories, Beyond Standard Model, CP violation, Solitons Monopoles and Instantons

ARXIV EPRINT: 1710.04213 


\section{Contents}

1 Introduction 1

2 Non-perturbative effects in product gauge groups 3

$2.1 \mathrm{SU}(3) \times \mathrm{SU}(3)$ model 3

$\begin{array}{lll}2.2 & \mathrm{SU}(3)^{N} \text { products } & 7\end{array}$

$\begin{array}{lll}3 & \text { UV sensitivity } & 9\end{array}$

$\begin{array}{lll}4 & \text { Phenomenology } & 10\end{array}$

$\begin{array}{lll}5 & \text { Conclusions } & 15\end{array}$

$\begin{array}{ll}\text { A Composite link fields } & 16\end{array}$

\section{Introduction}

The Standard Model (SM) describes physics remarkably well at the smallest scales probed so far. With the discovery of the Higgs boson, the SM can be consistently extrapolated to very high energies. However, there are a number of puzzles in the SM that remain. The strong CP problem is one such long standing problem. The QCD Lagrangian

$$
\mathcal{L}=-\frac{1}{4} G_{\mu \nu}^{a} G^{a, \mu \nu}-\frac{g^{2} \theta}{32 \pi^{2}} G_{\mu \nu}^{a} \tilde{G}^{a, \mu \nu}+\bar{q} M q
$$

in general violates $\mathrm{CP}$, with the $\mathrm{CP}$ violation encoded by

$$
\bar{\theta}=\theta+\arg \operatorname{det} M \text {. }
$$

Given that $\mathrm{CP}$ is badly broken by the weak interactions, we expect $\bar{\theta} \sim 1$. However, the QCD $\theta$-angle is constrained to be very small, $\bar{\theta} \lesssim 10^{-10}$, from limits on the electric dipole moment of the neutron $/ \mathrm{Hg}[1-3]$.

The solutions to the strong CP problem can be classified roughly into two categories. One class of solutions imposes discrete symmetries [4-6], like CP as in the Nelson-Barr mechanism [7-10] (see [11] for a recent discussion) or parity $(\mathrm{P})[9,12,13]$ that forbid the $\theta$-angle. In Nelson-Barr models there are generally no low-energy states, while parity models often require new light colored particles [14].

The second class of solutions invokes a global U(1) Peccei-Quinn symmetry [15, 16]. This $\mathrm{U}(1)_{P Q}$ has a mixed anomaly with QCD, such that QCD non-perturbative dynamics break PQ explicitly and the $\theta$-angle is dynamically relaxed to zero. The most economical version of such a solution is the massless (up-) quark solution [17], in which case the $\theta$-angle 
becomes unphysical (or equivalently is relaxed dynamically by the $\eta^{\prime}$ meson). However, lattice calculations indicate that all quarks are massive [18, 19], excluding the simplest model with a massless up-quark. Another elegant model that uses the PQ symmetry is the QCD axion [15, 16, 20, 21] (see [22] for a review). The $\mathrm{U}(1)_{P Q}$ is spontaneously broken in the UV, giving rise to a light pseudo-Nambu Goldstone boson, the axion. The axion makes the $\theta$ angle dynamical, and has a potential with a minimum at $\bar{\theta}=0$ [23].

In our paper we focus on this axion solution to the strong $\mathrm{CP}$ problem. Given the QCD axion decay constant $f_{a}$, the mass of the axion is determined by QCD dynamics [20],

$$
m_{a, \mathrm{QCD}}^{2}=\frac{m_{u} m_{d}}{\left(m_{u}+m_{d}\right)^{2}} \frac{f_{\pi}^{2} m_{\pi}^{2}}{f_{a}^{2}} \simeq \frac{(75.5 \mathrm{MeV})^{4}}{f_{a}^{2}} \equiv \frac{\Lambda_{0}^{4}}{f_{a}^{2}}
$$

The axion couples to the standard model through dimension- 5 operators suppressed by $f_{a}$, and the precise mass relationship equation (1.3) has motivated many existing and future experimental efforts (see ref. [24] for a review). In this work, we study an extension of the QCD axion framework where instead of a single axion relaxing the vacuum to $\bar{\theta}=0$, two or more axions naturally cooperate to solve the strong $\mathrm{CP}$ problem. Each of these axions can have a mass much larger than the standard QCD axion, motivating searches for axions in the $\left(m_{a}, f_{a}\right)$ plane outside of the QCD axion window given by equation (1.3).

This multi-axion solution to the strong $\mathrm{CP}$ problem arises in models that extend the low-energy $\mathrm{SU}(3)_{c}$ gauge group to be the diagonal subgroup of a parent $\mathrm{SU}(3) \times \mathrm{SU}(3) \times \ldots$ product gauge group, which is broken down to $\mathrm{SU}(3)_{c}$ at some high scale $M$. All the SM quarks are taken to be charged under a single $\mathrm{SU}(3)$ factor of the parent gauge group, and we introduce an axion for each individual $\mathrm{SU}(3)$ factors that independently relaxes the corresponding $\theta$-angle to 0 . Each $\mathrm{SU}(3)$ factor of the parent gauge group is more strongly coupled than the diagonal $\mathrm{SU}(3)_{c}$ subgroup, and therefore the contributions to the axion potentials from UV instantons near the scale $M$ can be larger than the non-perturbative potential generated at low energies from QCD. Even for the smallest extension, $\mathrm{SU}(3) \times$ $\mathrm{SU}(3) \rightarrow \mathrm{SU}(3)_{c}$, we find that the two axions can have masses significantly larger than for the standard QCD axion. In a follow-up work, ref. [25], we will describe a related family of models where each quark generation is charged under a different $\mathrm{SU}(3)$ factor, and the strong CP problem can be solved without introducing any axions degrees of freedom in a spirit similar to the massless up quark solution.

Other mechanisms have been proposed that may increase the mass of the QCD axion. The models of refs. [26-30] also extend $\mathrm{SU}(3)_{c}$ to an $\mathrm{SU}(3) \times \mathrm{SU}(3)$ gauge group, but with QCD living in a single factor instead of the diagonal subgroup. In these models, a discrete $Z_{2}$ symmetry is introduced, requiring an entire mirror SM matter sector. In contrast, our mechanism does not involve any new matter in the $\mathrm{SU}(3)$ factors, and the only new lowenergy states are the axions directly responsible for relaxing $\bar{\theta}$. The models of refs. [31-36] introduce extra colored matter at an intermediate scale so that QCD runs back to strong coupling at a high scale $M$ where $\mathrm{SU}(3)_{c}$ is embedded in a $\mathrm{SU}(3+n)$ gauge group. However, the introduction of new colored matter generally leads to either new hierarchy problems for scalars or new CP phases for fermions, spoiling the solution to the strong CP problem [37]. 
In contrast, in our mechanism no new colored states are introduced below the scale $M$, and extra CP phases that would feed into the UV axion potential are naturally absent.

In section 2, we describe in detail the structure of the model and the calculation of the non-perturbative contributions to the axion potentials at the scale $M$. In section 3 , we discuss the sensitivity of the mechanism to higher dimensional operators and the connection to the electroweak hierarchy problem. In section 4, we describe the limits on these models from axion-like particle (ALP) searches.

\section{Non-perturbative effects in product gauge groups}

\section{$2.1 \quad \mathrm{SU}(3) \times \mathrm{SU}(3)$ model}

We start with a simple extension of the SM where the QCD gauge group emerges from Higgsing a product group

$$
\mathrm{SU}(3)_{1} \times \mathrm{SU}(3)_{2} \rightarrow \mathrm{SU}(3)_{c}
$$

at a scale $M \gg \mathrm{TeV}$. This a similar set-up to the renormalizable coloron models of [3841], although in our case the scales will be far out of reach of the LHC. The SM quarks are charged only under the $\mathrm{SU}(3)_{1}$ gauge factor, and there are no fermions charged under $\mathrm{SU}(3)_{2}$. We take the theory to have two spontaneously broken anomalous $\mathrm{U}(1)_{P Q}$ symmetries at scales $f_{i}>M$, giving an axion $a_{1,2}$ in each $\mathrm{SU}(3)$ sector:

$$
\begin{aligned}
\mathcal{L}= & -\frac{1}{4}\left(G_{1}\right)_{\mu \nu}^{a}\left(G_{1}\right)^{a, \mu \nu}+\frac{g_{s 1}^{2}}{32 \pi^{2}}\left(\frac{a_{1}}{f_{1}}-\theta_{1}\right)\left(\widetilde{G}_{1}\right)_{\mu \nu}^{a}\left(G_{1}\right)^{a, \mu \nu} \\
& -\frac{1}{4}\left(G_{2}\right)_{\mu \nu}^{a}\left(G_{2}\right)^{a, \mu \nu}+\frac{g_{s 2}^{2}}{32 \pi^{2}}\left(\frac{a_{2}}{f_{2}}-\theta_{2}\right)\left(\widetilde{G}_{2}\right)_{\mu \nu}^{a}\left(G_{2}\right)^{a, \mu \nu}
\end{aligned}
$$

The gauge couplings $g_{s 1,2}$ and theta angles $\theta_{1,2}$ are independent parameters not related to each other by any symmetries. The presence of two independent axion degrees of freedom will allow both physical $\theta$-angles to be dynamically removed. An additional cross-coupling term for the axions $\propto \frac{a_{1}}{f_{1}} \tilde{G}_{2} G_{2}$ can not be removed by a field redefinition. It leads to a mass-mixing between the axions, but does not destabilize the solution to the strong $\mathrm{CP}$ problem or significantly change the phenomenology of the light axion states, so we take it to vanish for simplicity. The UV couplings of $a_{1,2}$ to the electroweak topological terms $W \tilde{W}$ and $B \tilde{B}$ will only be relevant for the axion phenomenology, and will be discussed in section 4 .

The theory will match to the $\mathrm{SM}$ at a scale $M$ where the $\mathrm{SU}(3)_{1} \times \mathrm{SU}(3)_{2}$ gauge group is Higgsed to a diagonal $\mathrm{SU}(3)_{c}$. For simplicity, we take the dynamics to be a bifundamental scalar field $\Sigma_{12}:(3, \overline{3}, 1)$ of $\mathrm{SU}(3)_{1} \times \mathrm{SU}(3)_{2} \times \mathrm{U}(1)_{\Sigma}$ with a renormalizable scalar potential

$$
V_{\Sigma}=-m_{\Sigma}^{2} \operatorname{Tr}\left(\Sigma_{12} \Sigma_{12}^{\dagger}\right)+\frac{\lambda}{2}\left[\operatorname{Tr}\left(\Sigma_{12} \Sigma_{12}^{\dagger}\right)\right]^{2}+\frac{\kappa}{2} \operatorname{Tr}\left(\Sigma_{12} \Sigma_{12}^{\dagger} \Sigma_{12} \Sigma_{12}^{\dagger}\right)
$$

inducing a vev [38, 41-43]

$$
\langle\Sigma\rangle=\frac{m_{\Sigma}}{\sqrt{\kappa+3 \lambda}} \mathbb{I}_{3} \equiv \frac{f_{\Sigma}}{2} \mathbb{I}_{3}
$$


One combination of the $\mathrm{SU}(3)$ gauge bosons becomes massive with $M_{V}^{2} \equiv M^{2}=\left(g_{s_{1}}^{2}+\right.$ $\left.g_{s_{2}}^{2}\right) f_{\Sigma}^{2}$, and the unbroken $\mathrm{SU}(3)_{\mathrm{QCD}}$ gauge symmetry is given by

$$
G_{(\mathrm{SM})}^{\mu}=\cos \gamma G_{1}^{\mu}+\sin \gamma G_{2}^{\mu}, \quad \tan \gamma=g_{s_{1}} / g_{s_{2}}
$$

The additional $\mathrm{U}(1)_{\Sigma}$ gauge factor forbids a trilinear term in the potential, and the corresponding gauge boson absorbs the otherwise massless singlet Goldstone mode. ${ }^{1}$

We can integrate out the heavy degrees of freedom to match to the SM at this scale. The tree-level matching condition gives the couplings of the two SU(3) factors in terms of the standard model QCD coupling evaluated at the scale $M$,

$$
\frac{1}{\alpha_{s}(\mu)}=\frac{1}{\alpha_{s_{1}}(\mu)}+\frac{1}{\alpha_{s_{2}}(\mu)}, \quad \mu=M,
$$

with $\alpha=g^{2} / 4 \pi$. Each individual gauge factor is more strongly coupled than the SM QCD gauge coupling, and this will lead to enhanced non-perturbative effects compared to the SM. The SM coupling $g_{s}(M)$ can be obtained with the 1-loop running from the top pole, $\alpha_{s}\left(m_{t}\right)=0.10$.

The tree-level value of the SM theta term is simply

$$
\bar{\theta}_{S M}=\bar{\theta}_{1}+\bar{\theta}_{2}
$$

Because the flavor symmetries are the same as the SM, the loop-level thresholds to $\bar{\theta}$ proportional to the CKM phase are negligible [44, 45].

Since we are integrating out the heavy degrees of freedom, we must also consider short distance non-perturbative effects in the individual $\mathrm{SU}(3)_{1}$ and $\mathrm{SU}(3)_{2}$ factors, which will generate the shift-symmetry breaking potentials for the axions. We will be interested in the regime where each factor is still at relatively weak coupling near $M$, and therefore the dilute instanton gas approximation (DGA) gives a good approximation for the non-perturbative effects. The effective Lagrangian generated for the axions is [46-48]

$$
\mathcal{L}_{a}=\Lambda_{1}^{4} \cos \left(\frac{a_{1}}{f_{1}}-\bar{\theta}_{1}\right)+\Lambda_{2}^{4} \cos \left(\frac{a_{2}}{f_{2}}-\bar{\theta}_{2}\right)+\frac{g_{s}^{2}}{32 \pi^{2}}\left(\left(\frac{a_{1}}{f_{1}}-\bar{\theta}_{1}\right)+\left(\frac{a_{2}}{f_{2}}-\bar{\theta}_{2}\right)\right) G \tilde{G}
$$

The axions still couple to the low energy QCD $\tilde{G} G$ term, and at the scale $\Lambda_{\mathrm{QCD}} \sim 1 \mathrm{GeV}$ the non-perturbative SM contributions to the axion potentials will be generated. However, unlike the standard axion case, the axion potential can be dominated by the higher energy contributions to the potential with scales $\Lambda_{1}$ and $\Lambda_{2}$ generated at the scale $M$. Although these scales $\Lambda_{1}$ and $\Lambda_{2}$ will be suppressed by non-perturbative and chiral suppression factors, they can still greatly exceed the scale of the standard axion potential.

\footnotetext{
${ }^{1}$ As an alternative to gauging the extra $\mathrm{U}(1)$ factor, the trilinear term $\mu \operatorname{Det} \Sigma_{12}+$ h.c could be introduced in the potential to lift the singlet Goldstone mode. Note that this CP phase in the trilinear term can be rotated away into the $\Sigma$ field, and the potential dynamically prefers a CP preserving $\Sigma$ vev [41].
} 
Crucially, and as is clear from the UV PQ symmetries of the theory, the new shortdistance non-perturbative contributions to the axion potentials are exactly aligned to remove the effective $\theta$-angle,

$$
\bar{\theta}_{\text {eff }}=\left\langle\left(\frac{a_{1}}{f_{1}}+\bar{\theta}_{1}\right)+\left(\frac{a_{2}}{f_{2}}+\bar{\theta}_{2}\right)\right\rangle=0 .
$$

The low-energy vacuum is in-fact guaranteed to align with $\bar{\theta}=0$ by a generalization of the Vafa-Witten argument [23]. This result is very different from theories which have new perturbative breakings of the PQ symmetry in the UV- for example, higher-dimensional operators violating the PQ symmetry give axion potentials that are generically misaligned and would spoil the axion solution to the strong CP problem [49-51].

We can now estimate how the mass of the two axions is affected by these UV nonperturbative contributions to the potential. In the $\mathrm{SU}(3)_{2}$ factor, where no colored fermions are present, the axion potential is suppressed only by the non-perturbative instanton action. The scale can be calculated as [46-48]

$$
\Lambda_{2}^{4}=\int_{\rho=0}^{\rho=1 / M} 2 \frac{d \rho}{\rho^{5}} D\left[\alpha_{s_{2}}(1 / \rho)\right]
$$

where the dimensionless instanton density depends non-perturbatively on the running gauge coupling as

$$
D[\alpha]=0.1\left(\frac{2 \pi}{\alpha}\right)^{6} e^{-\frac{2 \pi}{\alpha}}
$$

with $\alpha(1 / \rho)$ the running coupling evaluated at the scale $\mu=\rho^{-1}$ corresponding to the size of the instanton. Higher order corrections to the instanton density have been calculated in ref. [19]. In this work, we use the leading order result, and estimate the theoretical uncertainty by varying the scale at which the density is evaluated: $D\left[\alpha_{s}(1 / \rho)\right] \rightarrow$ $\left(D\left[\alpha_{s}(2 / \rho)\right], D\left[\alpha_{s}\left(\frac{1}{2 \rho}\right)\right]\right)$.

Since the instanton contribution dominantly comes from the IR, near the scale $M$, the instanton density in the integrand (2.9) can be approximated as

$$
D\left[\alpha_{s_{1,2}}(1 / \rho)\right] \approx D\left[\alpha_{s_{1,2}}(M)\right](\rho M)^{b_{1,2}},
$$

where the one-loop running coupling satisfies

$$
\frac{d \alpha_{s_{i}}^{-1}}{d \ln \mu}=\frac{b_{i}}{2 \pi}
$$

with $b_{1}=13 / 2$ and $b_{2}=21 / 2$ (including the contributions of the bifundamental scalar $\Sigma$ to the running). This gives

$$
\Lambda_{2}^{4}=\frac{4}{13} D\left[\alpha_{s_{2}}(M)\right] M^{4}
$$




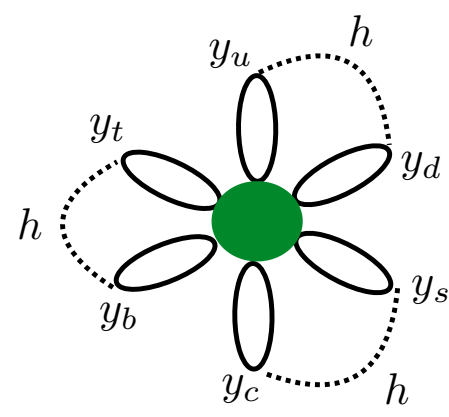

Figure 1. An instanton vacuum-diagram schematically generates a short distance contribution to the axion potential that is proportional to the breakings of the individual quark chiral U(1) factors by the Higgs Yukawa couplings. The dominant contribution at short distances is proportional to the Higgs vacuum fluctuations, corresponding to looping off the Higgs propagators.

In the $\mathrm{SU}(3)_{1}$ factor, there is a further suppression due to the Yukawa couplings and Higgs loops, as depicted in figure 1 , which can be estimated as $[35,36]$

$$
\Lambda_{1}^{4} \sim K \int_{\rho=0}^{\rho=1 / M} 2 \frac{d \rho}{\rho^{5}} D\left[\alpha_{s_{1}}(1 / \rho)\right] \approx K \frac{4}{5} D\left[\alpha_{s_{1}}(M)\right] M^{4}
$$

Where $K$ is a chiral suppression factor capturing the breaking of the $\mathrm{U}(1)^{6}$ axial symmetry of the individual quarks by the Yukawa couplings, ${ }^{2}$

$$
K=\left(\frac{y_{u}}{4 \pi}\right)\left(\frac{y_{d}}{4 \pi}\right)\left(\frac{y_{c}}{4 \pi}\right)\left(\frac{y_{s}}{4 \pi}\right)\left(\frac{y_{t}}{4 \pi}\right)\left(\frac{y_{b}}{4 \pi}\right) \approx 10^{-23} .
$$

We can compare the scales $\Lambda_{1,2}$ of the UV contributions to the scale $\Lambda_{0}$ of the usual nonperturbative axion potential generated around the QCD scale [20] given in equation (1.3).

The size of the non-perturbative UV potentials generated in each sector are shown in figure 2. The sensitivity to the large scale $M$ can overcome the non-perturbative and chiral suppression factors. The relation equation (2.6) between $\alpha_{s_{1}}$ and $\alpha_{s_{2}}$ implies that $\Lambda_{1}$ grows as $\Lambda_{2}$ shrinks. In much of the parameter range, $\Lambda_{1} \gg \Lambda_{0} \gg \Lambda_{2}$ or $\Lambda_{2} \gg \Lambda_{0} \gg \Lambda_{1}$. In these cases, one axion DOF will simply decouple, and the other will behave largely like the QCD axion.

The most interesting regime is instead when $\Lambda_{1}, \Lambda_{2} \gg \Lambda_{0}$ - in this case there will be no light state resembling the standard QCD axion. This regime generally corresponds to $\alpha_{s_{1}}>\alpha_{s_{2}}$ to compensate for the extra chiral suppression factor in $\Lambda_{1}$. As the scale $M$ increases, the scales $\Lambda_{1,2}$ grow — although the running of $\alpha_{3}$ to weak coupling at higher energies leads to stronger instanton suppression factors, this is overcome by the $M^{4}$ dependence of the potential. For $M \sim 10^{8} \mathrm{GeV}$, the UV effects can become comparable

\footnotetext{
${ }^{2}$ Ref. [35] considers the case where Yukawa couplings to a scalar state of mass $m_{s}$ break the chiral symmetries of the colored fermions in the theory. Their result contains an additional suppression factor of $m_{s}^{2} / M^{2}$, which would arise in theories where a scalar mass-insertion is necessary to violate some of the chiral symmetries, e.g. a two-higgs-doublet model. In contrast, in our case there are non-decoupling effects even in the limit $m_{s} \rightarrow 0$.
} 


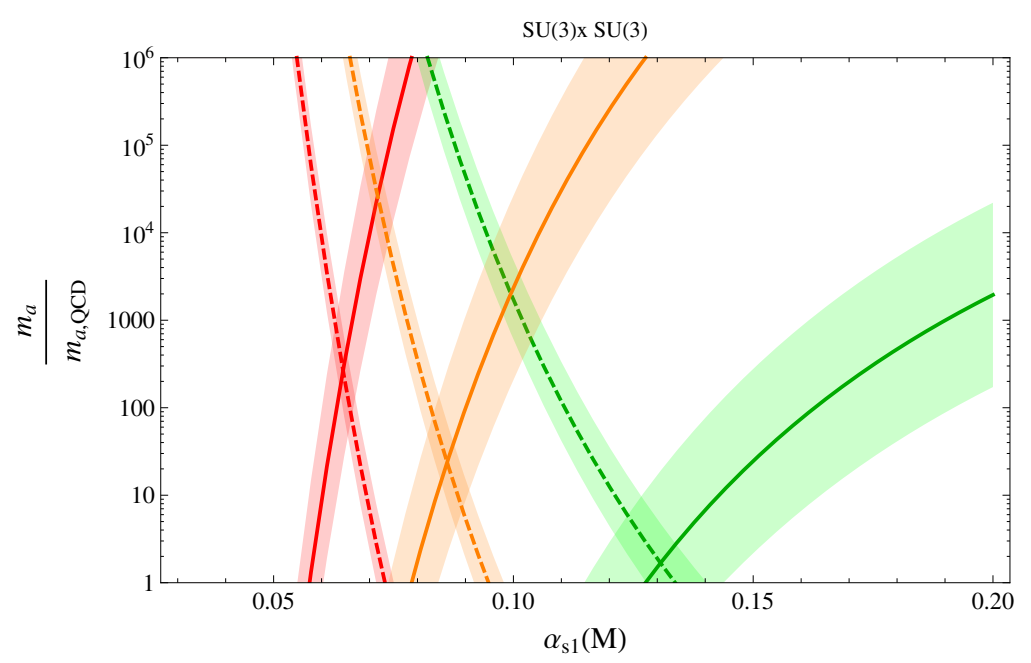

Figure 2. The scale of the UV contribution to the axion mass as a function of $\alpha_{s_{1}}$, compared to the mass $m_{a, Q C D}$ of a standard QCD axion given the same value of $f_{a}$. The solid lines give $m_{a_{1}}$ and the dashed lines give $m_{a_{2}}$, with $\alpha_{s_{2}}$ determined from $\alpha_{s_{1}}$ by the matching condition equation (2.6). The results are shown for a range of values of strong coupling scales, from left to right $M=10^{14} \mathrm{GeV}, 10^{11} \mathrm{GeV}, 10^{8} \mathrm{GeV}$ (red, orange, green). The parameter space of interest occurs when both $m_{a_{1}} / m_{a, Q C D} \gg 1$ and $m_{a_{2}} / m_{a, Q C D} \gg 1$ simultaneously, so that neither state has properties similar to the standard QCD axion.

in size to the IR potential, with $\Lambda_{1} \sim \Lambda_{2} \sim \Lambda_{0}$. For $M \sim 10^{14} \mathrm{GeV}$, effects as large as $\Lambda_{1} \sim \Lambda_{2} \sim 30 \Lambda_{0}$ can be realized. This realizes a model with two axions, each $\sim 1000$ times heavier than a standard QCD axion with the same decay constant $f$, with a potential aligned to dynamically set $\bar{\theta}=0$. Note that although the non-perturbative effects have a large effect on the axion masses in this regime, the individual gauge factors are still reasonably weakly coupled at the scale $M$, with $\alpha_{s_{1}}$ far away from the chiral-symmetry breaking phase which would be expected to occur at $\alpha_{s 1} \gtrsim 0.7-1[52,53]$.

So far we have considered the case $f_{a} \gtrsim M$ and assumed that all states in the spontaneous PQ-breaking sector are decoupled. If some of these states are lighter than $M$, the instanton effects can be suppressed. In a KSVZ axion model [54, 55] with $N_{f}$ flavors of vector-like quarks of mass $M_{\psi} \lesssim M$, there will be a suppression in the instanton calculation by a factor of $\sim\left(M_{\Psi} / \Lambda\right)^{N_{f}}$. We will take the benchmark $M_{\Psi}=f$ and $N_{f}=1$ for the phenomenological studies in section 4 , giving

$$
m_{a_{i}}^{2} \approx\left\{\begin{array}{ll}
\frac{\Lambda_{i}^{4}}{f_{a_{i}}^{2}} & f_{a_{i}}>M \\
\frac{\Lambda_{i}^{3}}{f_{a_{i}}} & f_{a_{i}}<M
\end{array} .\right.
$$

Other benchmarks like a KSVZ axion with $N_{f}>1$ or a DFSZ axion [56, 57] would typically have more severe suppressions for the regime $f_{a_{i}}<M$.

\section{$2.2 \mathrm{SU}(3)^{N}$ products}

Although each gauge factor in the $\mathrm{SU}(3) \times \mathrm{SU}(3)$ model is more strongly coupled than the $\mathrm{SM} \mathrm{SU}(3)_{\mathrm{QCD}}$, they remain relatively weakly coupled, and the additional short distance 


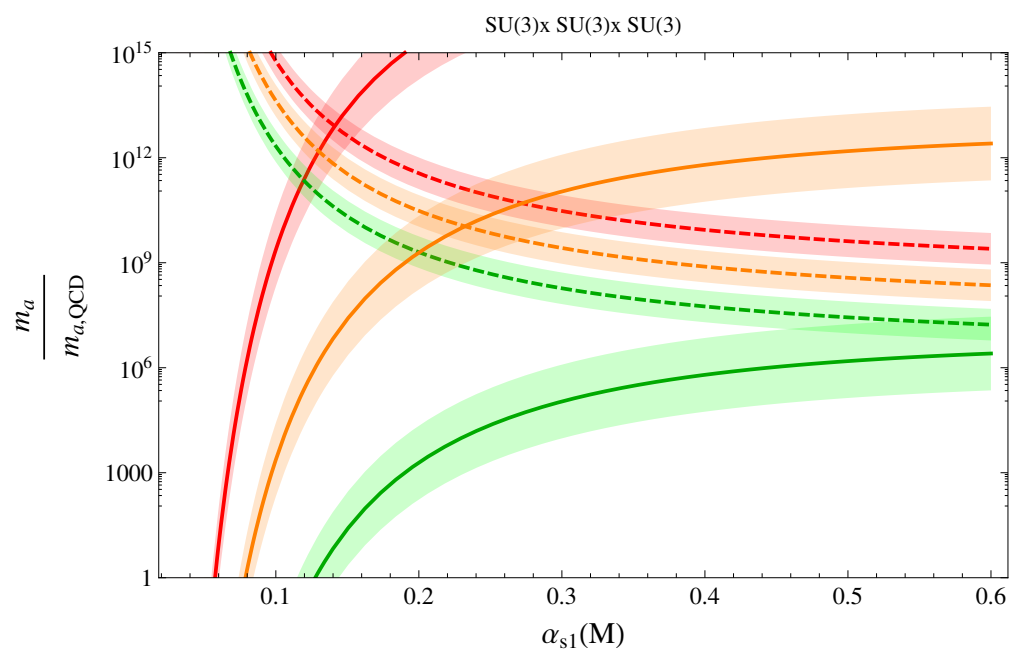

Figure 3. The scale of the UV contribution to the axion mass as a function of $\alpha_{s_{1}}$, compared to the mass $m_{a, Q C D}$ of a standard QCD axion given the same value of $f_{a}$. The solid lines give $m_{a_{1}}$ and the dashed lines give $m_{a_{2,3}}$, with $\alpha_{s_{2,3}}$ determined from $\alpha_{s_{1}}$ by the matching condition equation (2.6) taking $\alpha_{s_{2}}=\alpha_{s_{3}}$. The results are shown for a range of values of strong coupling scales, from top to bottom $M=10^{14} \mathrm{GeV}, 10^{11} \mathrm{GeV}, 10^{8} \mathrm{GeV}$ (red, orange, green). The parameter space of interest occurs when both $m_{a_{1}} / m_{a, Q C D} \gg 1$ and $m_{a_{2,3}} / m_{a, Q C D} \gg 1$ simultaneously, so that neither state has properties similar to the standard QCD axion.

non-perturbative effects still suffer a significant exponential suppression. Extending the gauge group to $\mathrm{SU}(3)^{N}$, with the $\mathrm{SU}(3)_{\mathrm{QCD}}$ emerging from the diagonal subgroup allows the gauge coupling in each individual factor to be substantially increased,

$$
\frac{1}{\alpha_{s}}(\mu)=\sum_{i=1}^{N} \frac{1}{\alpha_{s_{i}}(\mu)}, \quad \mu=M .
$$

The gauge group can be Higgsed to the diagonal subgroup at a scale $M$ by including multiple Higgs link fields $\Sigma_{12}, \ldots \Sigma_{N-1, N}$. An axion in each sector $a_{1}, \ldots, a_{N}$ can remove each $\theta_{1}, \ldots, \theta_{N}$. As for the $\mathrm{SU}(3) \times \mathrm{SU}(3)$ case, we take all of the SM quarks charged under $\mathrm{SU}(3)_{1}$, and assume no additional colored fermions are present in the other $\mathrm{SU}(3)$ factors. Even for $N=3$, the scale of the axion potential can now be dramatically increased compared to the standard QCD axion. Figure 3 shows the $N=3$ case taking $\alpha_{s_{2}}=\alpha_{s_{3}}$. For example for $M=10^{14} \mathrm{GeV}$, a model can be realized with 3 axions all with masses $\sim 10^{12}$ times larger than a standard QCD axion with the same decay constant $f$, again with a potential aligned to dynamically set $\bar{\theta}=0$.

It is interesting to ask how large the axion mass can be made if an even larger number of product factors are introduced. For $N \sim 10$, the couplings of the individual SU(3) factors can be made sufficiently strong to generate $\mathcal{O}(1)$ non-perturbative effects, giving $N-1$ axions with masses $m_{a_{i}} \sim M^{2} / f_{i}$, while the mass of the axion in the $\mathrm{SU}(3)_{1}$ sector will still be suppressed by the chiral suppression factor, giving $m_{a_{1}} \sim 10^{-12} M^{2} / f_{1}$. 


\section{UV sensitivity}

There are several interesting questions about the sensitivity of this multi-axion solution to UV physics above the scale $M$. First, as is familiar from the standard QCD axion picture, Planck-suppressed operators may spoil some of the PQ symmetries [49-51], naively leading to a misalignment of the axion potential by

$$
\Delta \bar{\theta} \sim \frac{f_{a}^{(d-2)}}{M_{\mathrm{pl}}^{(d-4)} m_{a}^{2}},
$$

with the dimension $d \geq 5$ of the operator depending on the details of the axion model. In our case, this problem is significantly alleviated compared to the standard QCD axion because smaller values of $f_{a}$ and larger values of $m_{a}$ can be realized. Figure 4 shows that large parts of parameter space remain open even assuming the most dangerous $d=5$ operators are present.

In our model there are also PQ-preserving higher dimensional operators that can be dangerous. Focusing for simplicity on the $\mathrm{SU}(3) \times \mathrm{SU}(3)$ case, at $D=6$ the operator

$$
\frac{\Sigma_{12} \tilde{G}_{2} \Sigma_{12}^{\dagger} G_{1}}{\Lambda_{\mathrm{UV}}^{2}}
$$

can introduce a shift $\Delta \bar{\theta} \sim M^{2} / \Lambda_{\mathrm{UV}}^{2}$ that is not canceled at the axion minima. Requiring $\bar{\theta} \lesssim 10^{-10}$ then requires a hierarchy $M \lesssim\left(10^{14} \mathrm{GeV}\right)\left(\frac{\Lambda_{\mathrm{UV}}}{M_{\mathrm{pl}}}\right)^{2}$.

Another possibility is that non-perturbative effects may not decouple sufficiently rapidly to protect the theory from $\mathrm{CP}$ violation at scales far above $M$. For example, if there are new unsuppressed sources of CP violation in the theory at the scale $\Lambda_{\mathrm{UV}}$, then non-perturbative effects at this scale can generate additional misaligned contributions to the axion potential. Because each gauge factor is asymptotically free, the nonperturbative effects decouple at high energies as $\alpha_{s_{i}}$ run to weak coupling. The $\mathrm{SU}(3)_{1}$ factor contains the SM quarks, and therefore runs to weak coupling the most slowly. From equations (2.14), (2.11), the scale of the potential generated for $a_{1}$ at $\Lambda_{\mathrm{UV}}$ is suppressed compared to the potential generated at $M$ by a factor

$$
\left(\frac{M}{\Lambda_{\mathrm{UV}}}\right)^{b_{1}-4}=\left(\frac{M}{\Lambda_{\mathrm{UV}}}\right)^{5 / 2}
$$

Assuming an $\mathcal{O}(1)$ misalignment of the potential generated at $\Lambda_{\mathrm{UV}}$, the limit $\bar{\theta} \lesssim 10^{-10}$ requires a hierarchy $M \lesssim\left(10^{15} \mathrm{GeV}\right)\left(\frac{\Lambda_{\mathrm{UV}}}{M_{\mathrm{pl}}}\right)^{5 / 2}$.

Therefore without additional assumptions about the nature of $\mathrm{CP}$ violation at the scale $\Lambda_{\mathrm{UV}}$, there must be a substantial hierarchy in scales $M \ll \Lambda_{\mathrm{UV}}$ to protect against higher dimensional operators and sufficiently suppress non-perturbative effects. In the simple model we have presented, $M$ is set by an elementary scalar field $\Sigma_{12}$ breaking the gauge group, and it seems that maintaining this hierarchy reintroduces as severe of a tuning as the tuning in $\bar{\theta}$ we have set out to address. Two well-known solutions exist - this hierarchy can be protected by supersymmetry (SUSY) or by a technicolor-like mechanism. 
In a SUSY model, the scale $M$ can be protected if $m_{\text {soft }} \lesssim M$. When $m_{\text {soft }} \ll M$, the instanton-generated potential will be suppressed by a factor of at least $\left(m_{\text {soft }} / M\right)^{5}$ due to insertions of gaugino masses and the PQ-breaking $b H_{u} H_{d}$ soft terms [36], so it is desirable to stay in the regime $m_{\text {soft }} \sim M$. Since $m_{\text {soft }} \sim M \gg 100 \mathrm{GeV}$, it is not possible to protect the weak scale with SUSY in this model, but at least the hierarchy $M \ll \Lambda_{\mathrm{UV}}$ can be maintained. However, the soft SUSY breaking introduces new CP phases into the theory, and the alignment of the axion potential which gives $\bar{\theta}=0$ is no longer guaranteed. As is familiar from low-scale SUSY, we must assume that the soft terms are communicated in a way that does not introduce new CP violating phases (for a review, see ref. [58]), since these would lead radiatively to misalignment of the short-distance axion potential generated near $M$ (the standard QCD axion mechanism is similarly sensitive to the CP violation in the soft sector when $m_{\text {soft }} \lesssim 100 \mathrm{TeV}$ [59]). Ref. [11] points out a similar sensitivity to SUSY-breaking CP violation in Nelson-Barr models.

In a technicolor-like solution, the $\Sigma$ can be made a composite of elementary fermions under a new strong group, with the scale $M$ generated dynamically. This resembles asymptotically free completions of the dimensional deconstruction framework [60]. However, because new fermions charged under $\mathrm{SU}(3)_{1}$ and $\mathrm{SU}(3)_{2}$ will be introduced, we must take care that their additional chiral symmetries are broken in a way that does not suppress the non-perturbative axion potential or re-introduce new CP phases. An example of such a model is given in appendix A.

\section{Phenomenology}

Laboratory experiments, cosmology, astrophysics, beam dump, and collider experiments can all be sensitive probes of new light pseudoscalars, also known as axion-like particles. Their reach can be parameterized in the space of masses $m_{a}$ and the dimension- 5 couplings of the new states, which scale as $\frac{1}{f_{a}}$. Although our models contain multiple axion-like states, the direct couplings in-between these states are phenomenologically unimportant and we can treat the limits on each axion-like state independently.

Since we have not specified the UV details of the spontaneous PQ-breaking, the heavy axions in our models may realize dimension- 5 couplings to the electroweak gauge bosons $a W \tilde{W}, a B \tilde{B}$, and light fermions $\partial^{\mu} a\left(f^{\dagger} \gamma_{5} \gamma_{\mu} f\right)$ in addition to the gluon coupling $a \tilde{G} G$. When $m_{a}<m_{\eta^{\prime}} \sim \mathrm{GeV}$, these additional couplings to the mass eigenstates are inevitable at low energies since the axions mix with the $\eta^{\prime}, \eta$, and $\pi_{0}$ mesons [29], as in the standard QCD axion model [20,21,61]. When the axion is light enough that hadronic decay channels are closed, $m_{a} \lesssim 3 m_{\pi}$, the phenomenology is primarily determined by the low-energy axionphoton coupling, and this ALP case has been well studied.

For $m_{a} \gtrsim 1 \mathrm{GeV}$, hadronic decay channels open and typically dominate, and studies focused on an axion decaying dominantly to electroweak gauge bosons no longer apply. Limits depending on these couplings are even further weakened when UV couplings to the electroweak gauge bosons are absent, since the mixing-induced couplings become rapidly suppressed as QCD runs to weak coupling. 
To study the limits on our models, we take as a benchmark an effective low-energy photon coupling of

$$
\mathcal{L}_{a \gamma \gamma}=\frac{e^{2}}{32 \pi^{2}} \frac{a_{i}}{f_{i}} F \tilde{F}
$$

throughout the parameter space (for $m_{a} \gg m_{\eta^{\prime}}$, the photon coupling will be entirely due to the UV physics, while for $m_{a} \lesssim m_{\eta^{\prime}}$ it is a result of both the UV coupling and the mixing with the neutral mesons). We use this simplified approximation as a rough guide to the phenomenology and a more realistic treatment would generically include couplings to the $Z$ as well as to other SM particles.

We show existing limits and future projections for axion experiments as a function of $m_{a}, f_{a}$ in figure 4 . We also include two benchmark models in which the axions are lifted to masses much larger than the standard QCD axion.

The first benchmark model is the $\mathrm{SU}(3) \times \mathrm{SU}(3)$ model with $\alpha_{s_{1}}(M)=0.063$ and $M=10^{14} \mathrm{GeV}$. This model contains two axions, each corresponding to a separate curve in the $\left(m_{a}, f_{a}\right)$ plane. In principle, $f_{1}$ and $f_{2}$ can occur at very disparate scales, so each axion could appear at any point on its curve, although it might be appealing to connect the scales $f_{1}$ and $f_{2}$ in a detailed model of the spontaneous PQ-breaking sector. At small values of $f_{a}$, both $\Lambda_{1}$ and $\Lambda_{2}$ become less than $\Lambda_{0}$. In this region one linear combination behaves as the usual QCD axion, and the orthogonal combination is light and has suppressed couplings to QCD.

The second benchmark model is the $\mathrm{SU}(3) \times \mathrm{SU}(3) \times \mathrm{SU}(3)$ model with $\alpha_{s_{1}}(M)=0.1$, $\alpha_{s_{2}}=\alpha_{s_{3}}$, and $M=10^{14} \mathrm{GeV}$. Of particular interest is a new region in parameter space for a $f_{a} \sim \mathrm{TeV}$ weak-scale axion that appears to be open in this model when $m_{a} \gtrsim \mathrm{GeV}$ - it would be interesting to study in further detail the limits on such a hadronically decaying state.

We have chosen these two specific benchmarks to illustrate the parameter space and the correlations between the scales of the multiple axions present in each model. We note however, that the parameter space of these models motivates axions spanning the entire $\left(m_{a}, f_{a}\right)$ plane above the standard QCD axion line.

We briefly describe the constraints and projections shown in figure 4. Current constraints are shown as shaded regions. The light blue shaded region are independent of a dark matter interpretation of the axion or its cosmology. The yellow shaded regions assume that the reheating temperature was large enough to have thermally produced axions, and that there was no large entropy dump to the SM after axion decoupling. The red shaded regions assume that the axion makes up all of the dark matter.

Misalignment and $\boldsymbol{\Omega}_{\mathrm{DM}}$. In parts of our parameter space, axions can make up all of the dark matter. In our model the mass of the axion goes to its zero temperature value much earlier than the time it starts oscillating, making the computation of relic abundance much simpler than the standard QCD axion case. In our case the relic abundance for a 
single axion with mass $m_{a}$ and decay constant $f_{a}$ is given as,

$$
\Omega_{a} h^{2}=\frac{m_{a}^{2} f_{a}^{2} \theta_{0}^{2}}{\rho_{\text {crit }} / h^{2}}\left(\frac{a\left(T_{f}\right)}{a_{0}}\right)^{3} \simeq 0.12\left(\frac{f_{a}}{10^{12} \mathrm{GeV}}\right)^{2}\left(\frac{m_{a}}{0.01 \mathrm{eV}}\right)^{1 / 2} \theta_{0}^{2}
$$

where $a\left(T_{f}\right)$ is the scale factor at the temperature $T_{f}$ where the axion starts oscillating, $H\left(T_{f}\right) \simeq m_{a} / 3, \theta_{0}$ is the initial misalignment angle, and $\rho_{\text {crit }}=3.96 \times 10^{-47} \mathrm{GeV}^{4}$ is the critical density of the Universe. This estimate ignores the thermal production of axions, which can become important for higher masses as well. We show the part of the parameter space in our benchmarks where heavy axions could make up the dark matter by solid lines in figure 4. It is interesting to note that in our models there is a new region of the $m_{a}-f_{a}$ parameter space where axions solve the strong $\mathrm{CP}$ problem and constitute all of the dark matter for generic initial conditions $\left(\theta_{0} \sim 1\right)$.

Cosmology constraints. The yellow region is ruled out by a combination of cosmological constraints, assuming that the reheat temperature was sufficiently high to produce an equilibrium thermal population of axions [62] which dominates over misalignment production in this region [63]. Future CMB observations can also put significant constraints on thermally produced axions from measurements of $\Delta N_{\text {eff }}$ that constrains extra relativistic degrees of freedom present during recombination. We show the sensitivity of the CMB-S4 assuming a reheat temperature of $T_{R}=10^{10} \mathrm{GeV}$ [64] with a horizontal gray line.

If the axion makes up dark matter, then its early universe values can be large enough to affect BBN [65]. This bound is superseded by the solar bound mentioned below.

Astrophysical bounds. Some of the strongest constraints on the axion parameter space arise from stellar physics, where axion coupling to photons is constrained by evolution of horizontal branch stars. For lower masses of axions supernova constraints and constraints from conversion of X-ray photons to axions in cluster magnetic fields [66-68] impose a stronger bound. Axion helioscopes such as CAST [69] also put constraints on the couplings of axions with photons, with the future IAXO helioscope [70] improving the reach. The light blue region includes these astrophysical constraints (adapted from [71]). We note that these astrophysical bounds off the QCD line are usually computed only for axion-like particles, i.e. with no nucleon coupling included. It will be interesting to recast these limits to the case where the axion does have a coupling to nucleons.

Axions which lie above the QCD line in figure 4 need a tuned negative contribution to their mass to cancel the partially cancel the mass from QCD. Their potential can then flip its sign due to finite density effects in astrophysical objects, which then source the axion field. This observation was used by ref. [72] to put bounds on this parameter space by dense astrophysical objects sourcing axion fields. In figure 4 we show the current bounds arising from the Sun and projections from neutron star observations (purple), which may be able to probe most of this tuned region of parameter space. We use $m_{a}=0.1 m_{a, Q C D}$ as a conservative projection from LIGO. Black hole superradiance [73] puts constraints on very light axions. 


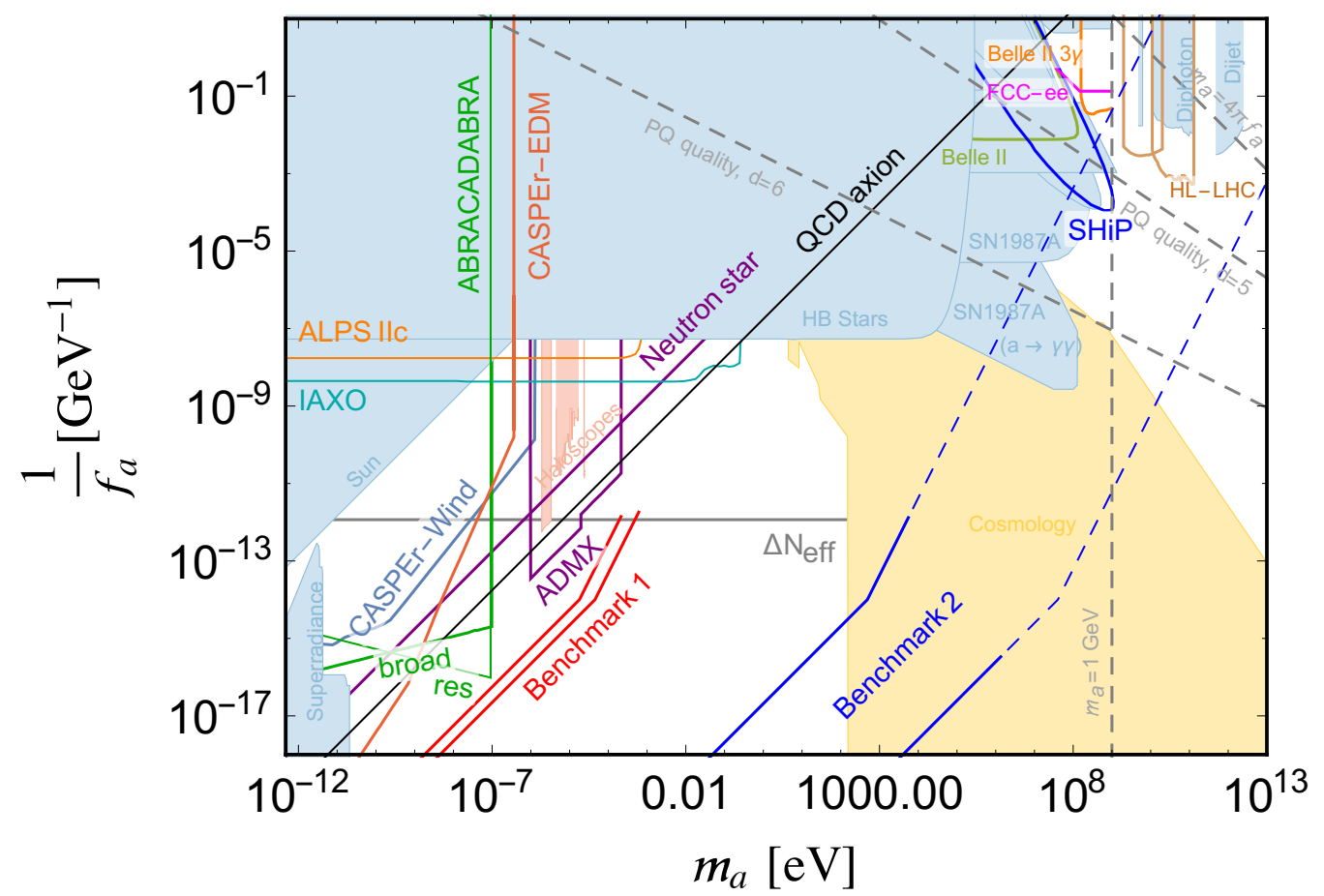

Figure 4. Limits on axions in the $\left(m_{a}, f_{a}\right)$ plane for an axion with couplings to gluons and photons given by equations (2.7) and (4.1). The black solid line is the standard QCD axion, with mass given by equation (1.3). Parallel to this line from left to right are the lines for $a_{1}$ and $a_{2}$ (red) in the $\mathrm{SU}(3) \times \mathrm{SU}(3)$ benchmark parameters described in the text, and $a_{2,3}$ and $a_{1}$ (blue) for the $\mathrm{SU}(3) \times \mathrm{SU}(3) \times \mathrm{SU}(3)$ benchmark parameters. The solid segments on these lines indicate where they could be realized as dark matter from the misalignment mechanism. The shaded regions show current constraints, and the regions bounded by solid lines are sensitivities of future experiments (see text for details).

Collider constraints. The possibility of heavier QCD axions also opens up weak-scale decay constants, making these axions visible at colliders. We show constraints from monojet searches at the LHC [74] and from LHC dijet [75-77] and diphoton searches [78-82] for pseudoscalar states produced in gluon fusion [83]. Also shown are the constraints from by monophoton and beam dump experiments (adapted from [84]). The Belle II experiment [85] will be sensitive to heavier axion-like particles at the weak scale [84]. The SHiP beam dump experiment [86] can cover intermediate mass scale axions which are not yet excluded by current experiments. Future $e^{+} e^{-}$colliders on the $Z$ mass peak can also improve on axion couplings. We show projections [71] for the FCC-ee collider [87].

Some of the analyses above were carried out for axion-like particles which only have a coupling to photons, and hence do not directly apply to our case when decays to hadrons become important. As a crude fix we have cut off all such limits at $m_{a}=1 \mathrm{GeV}$. We have also included constraints from ref. [88] which do take a gluon as well as a photon coupling into account.

Laboratory experiments. Laboratory experiments such as the haloscope ADMX constrains the QCD axion in the region where it can be the dark matter. We show regions of 
parameter space that can be covered by future experiments. The upgraded cavity experiment ADMX2 [89] will cover a larger range of QCD axion masses with higher sensitivity. The CASPEr experiments [90-92] propose using NMR techniques to measure time-varying EDMs induced by the axion, which will be sensitive for low-mass axions. The ABRACADABRA [93] experiment can also cover the low mass axion parameter space for axionphoton couplings. These experiments all rely on the axion being all of dark matter. The upgraded light-shining-through-wall experiment ALPS II [94] will be sensitive to larger couplings of axion-like particles with the photon.

We have included in figure 4 some dashed gray lines motivated by theoretical considerations. The line in the upper right corner signals the invalidity of the effective axion theory when $m_{a} \gtrsim 4 \pi f_{a}$. The reduced sensitivity to quality of the PQ symmetry through Planck suppressed higher dimensional operators as described by equation (3.1) are also shown. In regions above these lines, generic dimension- 5 and -6 operators do not spoil the solution to the strong $\mathrm{CP}$ problem. The vertical dashed gray line at $m_{a}=1 \mathrm{GeV}$ shows the scale where hadronic decays begin to dominate and axion mixings with the neutral mesons begin to be suppressed.

A few general comments which follow from our survey of the phenomenology:

- In our plot, most of the current and future observations only probe the region above the QCD line. As noted above, this region is tuned such that there is a negative mass contribution to the axion, with a minimum which is highly aligned with the QCD minimum, and a size comparable to the QCD contribution. However, experiments which proble the axion coupling to photons can extend below the QCD line in our plot if the coupling of the axion to photons is much larger than the coupling assumed here (as in [95-97]). With such an enhanced couplings these experiments will also be sensitive to heavier QCD axions as considered in this paper.

- When hadronic channels are open, the branching ratio to photons is tiny. The collider signals of a heavy QCD axion therefore differ from the more-often studied case of ALPs which only have a photon coupling. Our work shows that the combination of gluon and photon couplings is very well motivated even for heavy collider-observable axions and deserve further study.

- We also note that the phenomenology of this realization of a heavy axion is substantially different from the phenomenology in a $Z_{2}$ heavy axion model, where the axion may decay to light mirror-sector particles [29], and is generically not expected to appear with multiple copies.

- A combination of heavy axions can make up the dark matter density through the misalignment mechanism in a new part of $m_{a}-f_{a}$ plane, motivating searches in this region. A resonance search strategy for laboratory experiments might be less optimal if the dark matter density is not dominantly stored in axions at one mass. 


\section{Conclusions}

We have described a novel mechanism for solving the strong CP problem. In the standard QCD axion mechanism, a spontaneously broken anomalous $\mathrm{U}(1)_{P Q}$ symmetry results in an axion with a potential generated by non-perturbative effects near the scale $\Lambda_{\mathrm{QCD}}$. In the absence of any UV sources of perturbative PQ violation, the strong CP problem is solved dynamically when the axion relaxes to its $\mathrm{CP}$ preserving minimum. In our mechanism, we embed $\mathrm{QCD}$ in a $\mathrm{SU}(3)^{N}$ product group at a scale $M \gg \Lambda_{\mathrm{QCD}}$, and use a separate spontaneously broken PQ symmetry in each individual SU(3) factor to dynamically relax each individual $\bar{\theta}$ angle. Because each individual $\mathrm{SU}(3)$ factor is more strongly coupled than the SM QCD at the scale $M$, the non-perturbative contributions to the axion potentials at the scale $M$ can be much larger than those generated near $\Lambda_{\mathrm{QCD}}$ for the standard QCD axion. After integrating out physics above the scale $M$, the theory is just the standard model with $N$ axions, each with a PQ-violating potential that arose from non-perturbative effects near the scale $M$. Although the PQ symmetries are explicitly violated in this lowenergy effective theory, the non-perturbative origin of the axion potentials in the full theory guarantees that the minimum relaxes the low-energy $\bar{\theta}$ angle while generating a mass for each axion that is much larger than the standard QCD axion mass relationship.

Our assumptions about the existence of spontaneously broken anomalous PQ symmetries are on the same footing as the standard QCD axion, and it is encouraging that in general a large number of axions are expected in string theory models [98]. In fact, in our model, the possibility of solving the strong CP problem with a small value of $f_{a}$ and large value of $m_{a}$ can resolve the problem of maintaining a sufficient PQ quality in the presence of quantum-gravity corrections that plague the standard axion solution [49-51].

The main obstacle to the model we have proposed is the conflict with solutions to the electroweak hierarchy problem. Solving the electroweak hierarchy problem in this class of model suggests $M \sim \mathrm{TeV}$, while generating large axion masses requires $M \gg \mathrm{TeV}$. While we have shown that it is possible to stabilize at least the hierarchy $M \ll M_{\mathrm{pl}}$, it requires further model building to ensure that additional sources of $\mathrm{CP}$ violation do not spoil the mechanism.

Phenomenologically, the model motivates exploring the whole $\left(m_{a}, f_{a}\right)$ range of axionlike particles, including the range $m_{a} \gtrsim 3 m_{\pi}$ where hadronic decays will dominate. While the model requires the existence of multiple heavy axion states, it would be difficult to directly verify their connection to the strong CP problem, as the precision mass-coupling relationships predicted for the standard QCD-axion are no longer realized. Because there is the possibility of decoupling the heavy axions to scales $m_{a} \gg M_{W}$, this work can also be viewed as an interesting new theoretical example of a completely decoupling solution to the strong CP problem.

We would like to thank Asimina Arvanitaki, Savas Dimopoulos, Bogdan Dobrescu, Roni Harnik, Anson Hook, Junwu (Curly) Huang, Gustavo Marques Tavares, Lisa Randall, Diego Redigolo, Filippo Sala and Raman Sundrum for encouragement, helpful conversations, and comments on the manuscript. This work was initiated under a street-lamp at the Aspen Center for Physics, which is supported by National Science Foundation grant 


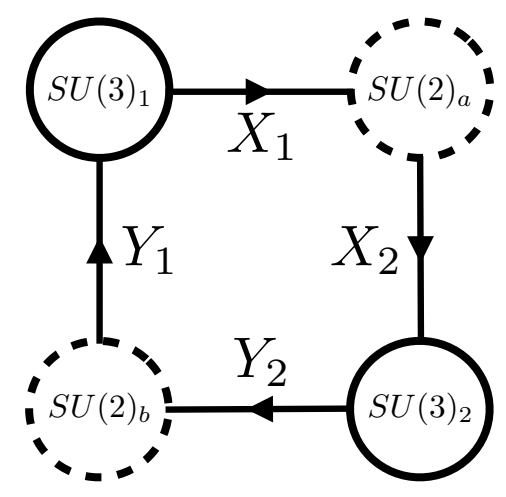

Figure 5. A moose model giving composite link fields to stabilize the hierarchy $M \ll \Lambda_{\mathrm{UV}}$.

PHY-1066293. PA is supported by the NSF grants PHY-0855591 and PHY-1216270. This manuscript has been authored by Fermi Research Alliance, LLC under Contract No. DEAC02-07CH11359 with the U.S. Department of Energy, Office of Science, Office of High Energy Physics. The United States Government retains and the publisher, by accepting the article for publication, acknowledges that the United States Government retains a nonexclusive, paid-up, irrevocable, world-wide license to publish or reproduce the published form of this manuscript, or allow others to do so, for United States Government purposes.

\section{A Composite link fields}

A model with composite link fields can stabilize the hierarchy $M \ll \Lambda_{\mathrm{UV}}$. For example, the $\mathrm{SU}(3)_{1} \times \mathrm{SU}(3)_{2}$ model can be extended to a $\mathrm{SU}(3)_{1} \times \mathrm{SU}(2)_{a} \times \mathrm{SU}(3)_{2} \times \mathrm{SU}(2)_{b}$ moose theory as shown in figure 5 , with condensation of elementary fermion bilinears $\left\langle X_{1} X_{2}\right\rangle$ and $\left\langle Y_{1} Y_{2}\right\rangle$ in the $\mathrm{SU}(2)$ factors breaking $\mathrm{SU}(3)_{1} \times \mathrm{SU}(3)_{2} \rightarrow \mathrm{SU}(3)_{c}$, making the scale $M$ dynamical.

In addition to the new gauge interactions, gauge invariant 4-fermion interactions of the form

$$
\mathcal{L}_{\chi}=\frac{\lambda_{1}}{M}\left(X_{1} X_{1}\right)\left(Y_{1} Y_{1}\right)+\frac{\lambda_{2}}{M}\left(X_{2} X_{2}\right)\left(Y_{2} Y_{2}\right)
$$

are necessary to break the additional chiral symmetries of the model. The instantons in $\mathrm{SU}(3)_{1}$ and $\mathrm{SU}(3)_{2}$ sectors will be suppressed by the coefficients $\lambda_{1}$ and $\lambda_{2}$, so this operator must either be generated at a nearby scale (without generating additional dangerous $\mathrm{CP}$ violating operators or a new hierarchy problem), or the theory must enter a conformal regime in the UV where these 4-fermion operators have large negative anomalous dimension.

Field redefinitions can be used to choose $\lambda_{1,2}$ real, and one remaining anomalous $\mathrm{U}(1)$ can be used to remove one combination of the $\theta$-angles in the $\mathrm{SU}(2)_{a}$ and $\mathrm{SU}(2)_{b}$ sectors. The remaining new $\theta$ angle may be removed by coupling an additional axion degree of freedom to the $\mathrm{SU}(2)_{a}$ or $\mathrm{SU}(2)_{b}$ sector, which will obtain a large mass $\sim \lambda_{1} \lambda_{2} M^{2} / f$. 
Open Access. This article is distributed under the terms of the Creative Commons Attribution License (CC-BY 4.0), which permits any use, distribution and reproduction in any medium, provided the original author(s) and source are credited.

\section{References}

[1] C.A. Baker et al., An improved experimental limit on the electric dipole moment of the neutron, Phys. Rev. Lett. 97 (2006) 131801 [hep-ex/0602020] [INSPIRE].

[2] B. Graner, Y. Chen, E.G. Lindahl and B.R. Heckel, Reduced Limit on the Permanent Electric Dipole Moment of Hg199, Phys. Rev. Lett. 116 (2016) 161601 [Erratum ibid. 119 (2017) 119901] [arXiv: 1601.04339] [INSPIRE].

[3] J.M. Pendlebury et al., Revised experimental upper limit on the electric dipole moment of the neutron, Phys. Rev. D 92 (2015) 092003 [arXiv:1509.04411] [INSPIRE].

[4] M.A.B. Beg and H.S. Tsao, Strong P, T Noninvariances in a Superweak Theory, Phys. Rev. Lett. 41 (1978) 278 [INSPIRE].

[5] H. Georgi, A Model of Soft CP Violation, Hadronic J. 1 (1978) 155 [InSPIRE].

[6] R.N. Mohapatra and G. Senjanović, Natural Suppression of Strong $p$ and t Noninvariance, Phys. Lett. B 79 (1978) 283 [INSPIRE].

[7] A.E. Nelson, Naturally Weak CP-violation, Phys. Lett. B 136 (1984) 387 [INSPIRE].

[8] S.M. Barr, Solving the Strong CP Problem Without the Peccei-Quinn Symmetry, Phys. Rev. Lett. 53 (1984) 329 [INSPIRE].

[9] K.S. Babu and R.N. Mohapatra, A Solution to the Strong CP Problem Without an Axion, Phys. Rev. D 41 (1990) 1286 [inSPIRE].

[10] G. Hiller and M. Schmaltz, Solving the Strong CP Problem with Supersymmetry, Phys. Lett. B 514 (2001) 263 [hep-ph/0105254] [INSPIRE].

[11] M. Dine and P. Draper, Challenges for the Nelson-Barr Mechanism, JHEP 08 (2015) 132 [arXiv: 1506. 05433] [INSPIRE].

[12] S.M. Barr, D. Chang and G. Senjanović, Strong CP problem and parity, Phys. Rev. Lett. 67 (1991) 2765 [INSPIRE].

[13] R.N. Mohapatra and A. Rasin, A supersymmetric solution to CP problems, Phys. Rev. D 54 (1996) 5835 [hep-ph/9604445] [INSPIRE].

[14] R.T. D'Agnolo and A. Hook, Finding the Strong CP problem at the LHC, Phys. Lett. B 762 (2016) 421 [arXiv: 1507.00336] [INSPIRE].

[15] R.D. Peccei and H.R. Quinn, Some Aspects of Instantons, Nuovo Cim. A 41 (1977) 309 [INSPIRE].

[16] R.D. Peccei and H.R. Quinn, CP Conservation in the Presence of Instantons, Phys. Rev. Lett. 38 (1977) 1440 [INSPIRE].

[17] G. 't Hooft, Symmetry Breaking Through Bell-Jackiw Anomalies, Phys. Rev. Lett. 37 (1976) 8 [inSPIRE].

[18] S. Aoki et al., Review of lattice results concerning low-energy particle physics, Eur. Phys. J. C 77 (2017) 112 [arXiv:1607.00299] [INSPIRE]. 
[19] M. Dine, P. Draper and G. Festuccia, Instanton Effects in Three Flavor QCD, Phys. Rev. D 92 (2015) 054004 [arXiv: 1410.8505] [INSPIRE].

[20] S. Weinberg, A New Light Boson?, Phys. Rev. Lett. 40 (1978) 223 [InSPIRE].

[21] F. Wilczek, Problem of Strong $P$ and T Invariance in the Presence of Instantons, Phys. Rev. Lett. 40 (1978) 279 [INSPIRE].

[22] Particle Data Group collaboration, C. Patrignani et al., Review of Particle Physics, Chin. Phys. C 40 (2016) 100001 [InSPIRE].

[23] C. Vafa and E. Witten, Parity Conservation in QCD, Phys. Rev. Lett. 53 (1984) 535 [INSPIRE].

[24] P.W. Graham, I.G. Irastorza, S.K. Lamoreaux, A. Lindner and K.A. van Bibber, Experimental Searches for the Axion and Axion-Like Particles, Ann. Rev. Nucl. Part. Sci. 65 (2015) 485 [arXiv: 1602.00039] [INSPIRE].

[25] P. Agrawal and K. Howe, A Flavorful Factoring of the Strong CP Problem, arXiv: 1712.05803 [INSPIRE].

[26] V.A. Rubakov, Grand unification and heavy axion, JETP Lett. 65 (1997) 621 [hep-ph/9703409] [INSPIRE].

[27] Z. Berezhiani, L. Gianfagna and M. Giannotti, Strong CP problem and mirror world: The Weinberg-Wilczek axion revisited, Phys. Lett. B 500 (2001) 286 [hep-ph/0009290] [InSPIRE].

[28] A. Hook, Anomalous solutions to the strong CP problem, Phys. Rev. Lett. 114 (2015) 141801 [arXiv:1411.3325] [INSPIRE].

[29] H. Fukuda, K. Harigaya, M. Ibe and T.T. Yanagida, Model of visible QCD axion, Phys. Rev. D 92 (2015) 015021 [arXiv:1504.06084] [INSPIRE].

[30] S. Dimopoulos, A. Hook, J. Huang and G. Marques-Tavares, A collider observable QCD axion, JHEP 11 (2016) 052 [arXiv: 1606. 03097] [INSPIRE].

[31] B. Holdom and M.E. Peskin, Raising the Axion Mass, Nucl. Phys. B 208 (1982) 397 [INSPIRE].

[32] B. Holdom, Strong QCD at High-energies and a Heavy Axion, Phys. Lett. B 154 (1985) 316 [Erratum ibid. B 156 (1985) 452] [INSPIRE].

[33] M. Dine and N. Seiberg, String Theory and the Strong CP Problem, Nucl. Phys. B 273 (1986) 109 [INSPIRE].

[34] K. Choi, C.W. Kim and W.K. Sze, Mass Renormalization by Instantons and the Strong CP Problem, Phys. Rev. Lett. 61 (1988) 794 [inSPIRE].

[35] J.M. Flynn and L. Randall, A Computation of the Small Instanton Contribution to the Axion Potential, Nucl. Phys. B 293 (1987) 731 [InSPIRE].

[36] K. Choi and H.D. Kim, Small instanton contribution to the axion potential in supersymmetric models, Phys. Rev. D 59 (1999) 072001 [hep-ph/9809286] [INSPIRE].

[37] A. Albaid, M. Dine and P. Draper, Strong CP and SUZ, JHEP 12 (2015) 046 [arXiv: 1510.03392] [INSPIRE].

[38] Y. Bai and B.A. Dobrescu, Heavy octets and Tevatron signals with three or four b jets, JHEP 07 (2011) 100 [arXiv: 1012.5814] [INSPIRE]. 
[39] R.S. Chivukula, A. Farzinnia, J. Ren and E.H. Simmons, Constraints on the Scalar Sector of the Renormalizable Coloron Model, Phys. Rev. D 88 (2013) 075020 [Erratum ibid. D 89 (2014) 059905] [arXiv: 1307.1064] [INSPIRE].

[40] R.S. Chivukula, A. Farzinnia and E.H. Simmons, Vacuum Stability and Triviality Analyses of the Renormalizable Coloron Model, Phys. Rev. D 92 (2015) 055002 [arXiv:1504.03012] [INSPIRE].

[41] Y. Bai and B.A. Dobrescu, Minimal SU(3) $\times$ SU(3) Symmetry Breaking Patterns, Phys. Rev. D 97 (2018) 055024 [arXiv:1710.01456] [INSPIRE].

[42] E. Nardi, Naturally large Yukawa hierarchies, Phys. Rev. D 84 (2011) 036008 [arXiv: 1105.1770] [INSPIRE].

[43] J.R. Espinosa, C.S. Fong and E. Nardi, Yukawa hierarchies from spontaneous breaking of the $\mathrm{SU}(3)_{L} \times \mathrm{SU}(3)_{R}$ flavour symmetry?, JHEP 02 (2013) 137 [arXiv: 1211.6428] [INSPIRE].

[44] J.R. Ellis and M.K. Gaillard, Strong and Weak CP-violation, Nucl. Phys. B 150 (1979) 141 [INSPIRE].

[45] M. Dugan, B. Grinstein and L.J. Hall, CP Violation in the Minimal $N=1$ Supergravity Theory, Nucl. Phys. B 255 (1985) 413 [inSPIRE].

[46] G. 't Hooft, Computation of the Quantum Effects Due to a Four-Dimensional Pseudoparticle, Phys. Rev. D 14 (1976) 3432 [Erratum ibid. D 18 (1978) 2199] [InSPIRE].

[47] C.G. Callan Jr., R.F. Dashen and D.J. Gross, Toward a Theory of the Strong Interactions, Phys. Rev. D 17 (1978) 2717 [InSPIRE].

[48] N. Andrei and D.J. Gross, The Effect of Instantons on the Short Distance Structure of Hadronic Currents, Phys. Rev. D 18 (1978) 468 [INSPIRE].

[49] S.M. Barr and D. Seckel, Planck scale corrections to axion models, Phys. Rev. D 46 (1992) 539 [INSPIRE].

[50] M. Kamionkowski and J. March-Russell, Planck scale physics and the Peccei-Quinn mechanism, Phys. Lett. B 282 (1992) 137 [hep-th/9202003] [INSPIRE].

[51] S. Ghigna, M. Lusignoli and M. Roncadelli, Instability of the invisible axion, Phys. Lett. B 283 (1992) 278.

[52] C.D. Roberts and A.G. Williams, Dyson-Schwinger equations and their application to hadronic physics, Prog. Part. Nucl. Phys. 33 (1994) 477 [hep-ph/9403224] [INSPIRE].

[53] T. Appelquist, A. Nyffeler and S.B. Selipsky, Analyzing chiral symmetry breaking in supersymmetric gauge theories, Phys. Lett. B 425 (1998) 300 [hep-th/9709177] [INSPIRE].

[54] J.E. Kim, Weak Interaction Singlet and Strong CP Invariance, Phys. Rev. Lett. 43 (1979) 103 [INSPIRE].

[55] M.A. Shifman, A.I. Vainshtein and V.I. Zakharov, Can Confinement Ensure Natural CP Invariance of Strong Interactions?, Nucl. Phys. B 166 (1980) 493 [InSPIRE].

[56] M. Dine, W. Fischler and M. Srednicki, A Simple Solution to the Strong CP Problem with a Harmless Axion, Phys. Lett. B 104 (1981) 199 [INSPIRE].

[57] A.R. Zhitnitsky, On Possible Suppression of the Axion Hadron Interactions (in Russian), Sov. J. Nucl. Phys. 31 (1980) 260 [Yad. Fiz. 31 (1980) 497] [INSPIRE]. 
[58] S.P. Martin, A supersymmetry primer, Adv. Ser. Direct. High Energy Phys. 18 (1998) 1 [hep-ph/9709356] [INSPIRE].

[59] C. Hamzaoui and M. Pospelov, The limits on CP odd four fermion operators containing strange quark field, Phys. Rev. D 60 (1999) 036003 [hep-ph/9901363] [INSPIRE].

[60] N. Arkani-Hamed, A.G. Cohen and H. Georgi, (De)constructing dimensions, Phys. Rev. Lett. 86 (2001) 4757 [hep-th/0104005] [INSPIRE].

[61] G. Grilli di Cortona, E. Hardy, J. Pardo Vega and G. Villadoro, The QCD axion, precisely, JHEP 01 (2016) 034 [arXiv: 1511.02867] [INSPIRE].

[62] D. Cadamuro and J. Redondo, Cosmological bounds on pseudo Nambu-Goldstone bosons, JCAP 02 (2012) 032 [arXiv:1110.2895] [INSPIRE].

[63] P. Arias, D. Cadamuro, M. Goodsell, J. Jaeckel, J. Redondo and A. Ringwald, WISPy Cold Dark Matter, JCAP 06 (2012) 013 [arXiv:1201.5902] [INSPIRE].

[64] D. Baumann, D. Green and B. Wallisch, New Target for Cosmic Axion Searches, Phys. Rev. Lett. 117 (2016) 171301 [arXiv: 1604.08614] [INSPIRE].

[65] K. Blum, R.T. D'Agnolo, M. Lisanti and B.R. Safdi, Constraining Axion Dark Matter with Big Bang Nucleosynthesis, Phys. Lett. B 737 (2014) 30 [arXiv:1401.6460] [InSPIRE].

[66] M. Berg et al., Constraints on Axion-Like Particles from X-ray Observations of NGC1275, Astrophys. J. 847 (2017) 101 [arXiv: 1605.01043] [INSPIRE].

[67] M.C.D. Marsh, H.R. Russell, A.C. Fabian, B.P. McNamara, P. Nulsen and C.S. Reynolds, A New Bound on Axion-Like Particles, JCAP 12 (2017) 036 [arXiv:1703.07354] [InSPIRE].

[68] J.P. Conlon, F. Day, N. Jennings, S. Krippendorf and M. Rummel, Constraints on Axion-Like Particles from Non-Observation of Spectral Modulations for X-ray Point Sources, JCAP 07 (2017) 005 [arXiv: 1704.05256] [INSPIRE].

[69] CAST collaboration, V. Anastassopoulos et al., New CAST Limit on the Axion-Photon Interaction, Nature Phys. 13 (2017) 584 [arXiv:1705.02290] [INSPIRE].

[70] J.K. Vogel et al., IAXO - The International Axion Observatory, in 8th Patras Workshop on Axions, WIMPs and WISPs (AXION-WIMP 2012) Chicago, Illinois, July 18-22, 2012, arXiv: 1302.3273 fermilab-pub-13-699 (2013).

[71] J. Jaeckel and M. Spannowsky, Probing MeV to $90 \mathrm{GeV}$ axion-like particles with LEP and LHC, Phys. Lett. B 753 (2016) 482 [arXiv:1509.00476] [INSPIRE].

[72] A. Hook and J. Huang, Probing axions with neutron star inspirals and other stellar processes, JHEP 06 (2018) 036 [arXiv:1708.08464] [INSPIRE].

[73] A. Arvanitaki, M. Baryakhtar and X. Huang, Discovering the QCD Axion with Black Holes and Gravitational Waves, Phys. Rev. D 91 (2015) 084011 [arXiv:1411.2263] [INSPIRE].

[74] K. Mimasu and V. Sanz, ALPs at Colliders, JHEP 06 (2015) 173 [arXiv:1409.4792] [INSPIRE].

[75] CMS collaboration, Search for low mass vector resonances decaying into quark-antiquark pairs in proton-proton collisions at $\sqrt{s}=13 \mathrm{TeV}, \mathrm{JHEP} 01$ (2018) 097 [arXiv:1710.00159] [INSPIRE].

[76] ATLAS collaboration, Search for new phenomena in dijet events using $37 \mathrm{fb}^{-1}$ of $p p$ collision data collected at $\sqrt{s}=13$ TeV with the ATLAS detector, Phys. Rev. D 96 (2017) 052004 [arXiv: 1703.09127] [INSPIRE]. 
[77] CMS collaboration, Searches for dijet resonances in pp collisions at $\sqrt{s}=13$ TeV using data collected in 2016., CMS-PAS-EXO-16-056 (2016).

[78] ATLAS collaboration, Search for scalar diphoton resonances with $15.4 \mathrm{fb}^{-1}$ of data collected at $\sqrt{s}=13$ TeV in 2015 and 2016 with the ATLAS detector, ATLAS-CONF-2016-059 (2016).

[79] CMS collaboration, Search for high-mass diphoton resonances in proton-proton collisions at 13 TeV and combination with 8 TeV search, Phys. Lett. B 767 (2017) 147 [arXiv: 1609.02507] [INSPIRE].

[80] ATLAS collaboration, Search for Scalar Diphoton Resonances in the Mass Range 65-600 GeV with the ATLAS Detector in pp Collision Data at $\sqrt{s}=8 \mathrm{TeV}$, Phys. Rev. Lett. 113 (2014) 171801 [arXiv:1407.6583] [INSPIRE].

[81] CMS collaboration, Search for new resonances in the diphoton final state in the mass range between 80 and $110 \mathrm{GeV}$ in pp collisions at $\sqrt{s}=8 \mathrm{TeV}$, CMS-PAS-HIG-14-037 (2014).

[82] CMS collaboration, Search for new resonances in the diphoton final state in the mass range between 70 and $110 \mathrm{GeV}$ in pp collisions at $\sqrt{s}=8$ and 13 TeV, CMS-PAS-HIG-17-013 (2017).

[83] A. Belyaev et al., Di-boson signatures as Standard Candles for Partial Compositeness, JHEP 01 (2017) 094 [Erratum ibid. 12 (2017) 088] [arXiv:1610.06591] [INSPIRE].

[84] M.J. Dolan, T. Ferber, C. Hearty, F. Kahlhoefer and K. Schmidt-Hoberg, Revised constraints and Belle II sensitivity for visible and invisible axion-like particles, JHEP 12 (2017) 094 [arXiv: 1709.00009] [INSPIRE].

[85] Belle-II collaboration, T. Abe et al., Belle II Technical Design Report, arXiv:1011.0352 [INSPIRE].

[86] SHIP collaboration, M. Anelli et al., A facility to Search for Hidden Particles (SHiP) at the CERN SPS, arXiv: 1504.04956 [INSPIRE].

[87] Tlep Design Study Working Group collaboration, M. Bicer et al., First Look at the Physics Case of TLEP, JHEP 01 (2014) 164 [arXiv:1308.6176] [InSPIRE].

[88] A. Mariotti, D. Redigolo, F. Sala and K. Tobioka, New LHC bound on low-mass diphoton resonances, Phys. Lett. B 783 (2018) 13 [arXiv:1710.01743] [INSPIRE].

[89] T.M. Shokair et al., Future Directions in the Microwave Cavity Search for Dark Matter Axions, Int. J. Mod. Phys. A 29 (2014) 1443004 [arXiv:1405.3685] [InSPIRE].

[90] P.W. Graham and S. Rajendran, New Observables for Direct Detection of Axion Dark Matter, Phys. Rev. D 88 (2013) 035023 [arXiv:1306.6088] [INSPIRE].

[91] D. Budker, P.W. Graham, M. Ledbetter, S. Rajendran and A. Sushkov, Proposal for a Cosmic Axion Spin Precession Experiment (CASPEr), Phys. Rev. X 4 (2014) 021030 [arXiv: 1306.6089] [INSPIRE].

[92] A. Garcon et al., The Cosmic Axion Spin Precession Experiment (CASPEr): a dark-matter search with nuclear magnetic resonance, arXiv: 1707.05312 [INSPIRE].

[93] Y. Kahn, B.R. Safdi and J. Thaler, Broadband and Resonant Approaches to Axion Dark Matter Detection, Phys. Rev. Lett. 117 (2016) 141801 [arXiv:1602.01086] [INSPIRE].

[94] R. Bähre et al., Any light particle search II —Technical Design Report, 2013 JINST 8 T09001 [arXiv: 1302.5647] [INSPIRE]. 
[95] L. Di Luzio, F. Mescia and E. Nardi, Redefining the Axion Window, Phys. Rev. Lett. 118 (2017) 031801 [arXiv: 1610.07593] [INSPIRE].

[96] M. Farina, D. Pappadopulo, F. Rompineve and A. Tesi, The photo-philic QCD axion, JHEP 01 (2017) 095 [arXiv:1611.09855] [INSPIRE].

[97] P. Agrawal, J. Fan, M. Reece and L.-T. Wang, Experimental Targets for Photon Couplings of the QCD Axion, JHEP 02 (2018) 006 [arXiv: 1709.06085] [INSPIRE].

[98] A. Arvanitaki, S. Dimopoulos, S. Dubovsky, N. Kaloper and J. March-Russell, String Axiverse, Phys. Rev. D 81 (2010) 123530 [arXiv:0905.4720] [INSPIRE]. 